\title{
Guide to LIVING BIRDS
}

\author{
J. E. WEBB \\ Professor of Zoology, Westfield College, \\ University of London \\ J. A. WALLWORK \\ Reader in Zoology, Westfield College, \\ University of London \\ J. H. ELGOOD \\ Formerly Associate Professor of Zoology, \\ University of Ibadan, Nigeria
}

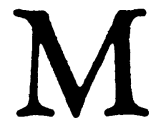


(c) J. E. Webb, J. A. Wallwork and J. H. Elgood 1979

All rights reserved. No part of this publication may be reproduced or transmitted, in any form or by any means, without permission

First published 1979 by

THE MACMILLAN PRESS LTD

London and Basingstoke

Associated companies in Delhi Dublin

Hong Kong Johannesburg Lagos Melbourne

New York Singapore and Tokyo

\section{British Library Cataloguing in Publication Data}

Webb, Joseph Ernest

Guide to living birds.

1. Birds - Classification

I. Title II. Wallwork, John Anthony

III. Elgood, John Hamel

598.2'01'2 QL677

ISBN 978-1-349-03615-8 ISBN 978-1-349-03613-4 (eBook)

DOI 10.1007/978-1-349-03613-4

This book is sold subject to the standard conditions of the Net Book Agreement

The paperback edition of this book is sold subject to the condition that it shall not, by way of trade or otherwise, be lent, resold, hired out, or otherwise circulated without the publisher's prior consent in any form of binding or cover other than that in which it is published and without a similar condition including this condition being imposed on the subsequent purchaser. 


\section{Preface}

This Guide to the Living Birds is one of a series covering between them the entire animal kingdom. Each guide uses an annotated classification to give a basic understanding of the animals under consideration, including their relationship with other animals and the interrelations within the group. Thus this book first views birds as highly specialised tetrapod vertebrates and then presents a pattern of their diversity.

Although the concept developed in all these guides is the same, they differ to some extent in their approach. In most groups of animals classification is based on structural features alone. But in birds their basic structure is so uniform (except for details often difficult to see) that ecological and behavioural differences must also be used. Birds have an immense popular appeal due largely to the ease with which they can be seen in the field. They are relatively large, colourful, diurnal animals that do things. They migrate, establish territory, court and breed and most have recognisable voices.

Moreover television cameras are now bringing many of them to our attention from remote parts so that the worldwide coverage of this guide is useful. We have been concerned, therefore, to present a reasoned, if slightly unorthodox, classification that should not offend the scientist, but will appeal to the amateur ornithologist.

Birds, like mammals, being comparative newcomers in evolutionary terms, have very interesting patterns of distribution, sometimes only explained by the movements of the continents in the past. (Continental drift has been dealt with at some length in the companion. volume on the reptiles.) A major feature of the guide, therefore, is the inclusion of maps showing the areas occupied by almost all the bird families that are not worldwide. Such maps are, of course, only approximate since birds 
continually change their range through migration, human introduction and re-introduction and Man's destruction of habitat.

There are so many excellent major works on birds that to present any sort of literature list would be virtually impossible in a small book. But a New Dictionary of Birds edited by A. Landsborough Thomson, Nelson, Edinburgh \& London, 1964 has provided the basis for the classification used. The popular (English) names used and the division of families into genera and species have been largely taken from A Checklist of the Birds of the World, E.S. Gruson, Collins, London, 1976. For a more detailed account of the structural and biological features of each family of birds Fundamentals of Ornithology, J. Van Tyne \& A.J. Berger, Dover, New York, 1959 is suggested, though this work follows a slightly different classification, especially in the arrangement of the song birds.

It is worth mentioning that this book is in no sense a 'Field Guide' of which there are many for the identification of birds from particular areas such as Britain, Europe, West Africa, North America. The Guide to the Living Birds is an organised summary for the student which, in addition, will give the answers to such questions as - how many kinds of kingfisher are there? - in what parts of the world are they found? - what are their nearest relatives? - do all have mainly blue plumage?

We are again grateful to Mrs. Margaret Clarke for the meticulous preparation of the typescript for photolithography and we are greatly indebted to Mr. Phil Brooks who made the drawings of the birds which are an integral part of the book. 


\section{Contents}

age

1

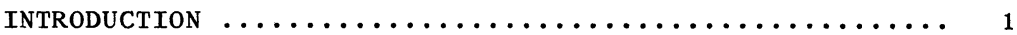

The uniformity of bird architecture $\ldots \ldots \ldots \ldots \ldots 2$

Problems of bird systematics .............. 2

The current phylogenetic classification of birds . 3

The systematic treatment used in this guide ..... 6

Geographical distribution ............... 9

Scientific names .................... 12

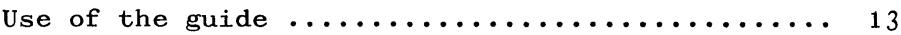

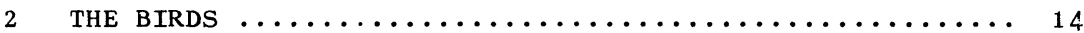

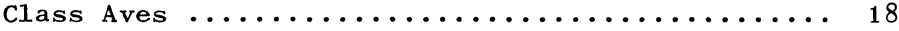

$\begin{array}{lllllllllllllllll}P & A & R & T & G & \text { G } & \text { U N D } & \text { B I R D S }\end{array}$

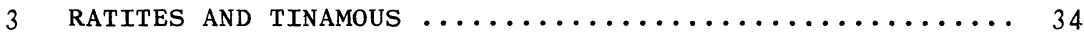

Superorder Ratitae ................. 36

Order Struthioniformes $\ldots \ldots \ldots \ldots \ldots \ldots \ldots \ldots, 41$

Family struthionidae ............ 41

Order Rheiformes ................. 42

Family Rheidae ............... 42

Order Casuariiformes ............... 43

Family Casuariidae ............... 44

Family Dromaiidae ............. 45

Order Apterygiformes $\ldots \ldots \ldots \ldots \ldots \ldots \ldots \ldots 46$

Family Apterygidae ............. 46

Superorder Tinamae ............... 47

Order Tinamiformes $\ldots \ldots \ldots \ldots \ldots \ldots \ldots \ldots, 47$

Family Tinamidae $\ldots \ldots \ldots \ldots \ldots \ldots \ldots \ldots \ldots$

4 GAME BIRDS, CRANES \& ALLIES $\ldots \ldots \ldots \ldots \ldots \ldots \ldots \ldots \ldots \ldots \ldots \ldots \ldots$

Superorder Carinatae ............... 51

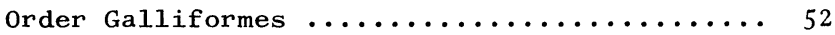

Suborder Galli .................... 52

Family Megapodidae .............. 54

Family Cracidae .................. 55 


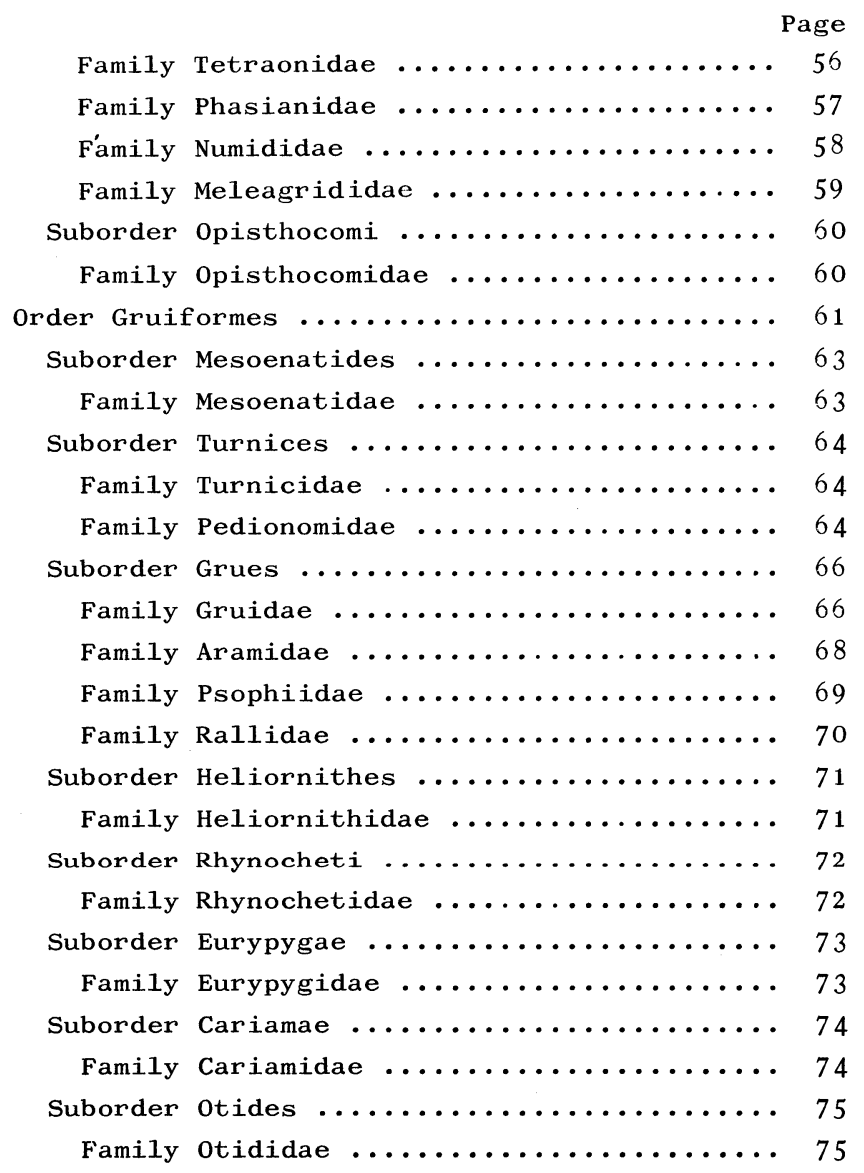

$\begin{array}{llllllllllllll}\text { P A R T II A } & \text { A U A T I C B I R D S }\end{array}$

$5 \quad$ SHORE BIRDS $\ldots \ldots \ldots \ldots \ldots \ldots \ldots \ldots \ldots \ldots \ldots \ldots \ldots \ldots \ldots \ldots \ldots \ldots$

Order Charadriiformes ................. 80

Suborder Charadrii .................. 81

Family Jacanidae ................. 82

Family Rostratulidae ............... 83

Family Haematopodidae ................ 84

Family Charadriidae .................... 85

Family Scolopacidae ............... 86

Family Recurvirostridae ............ 87 


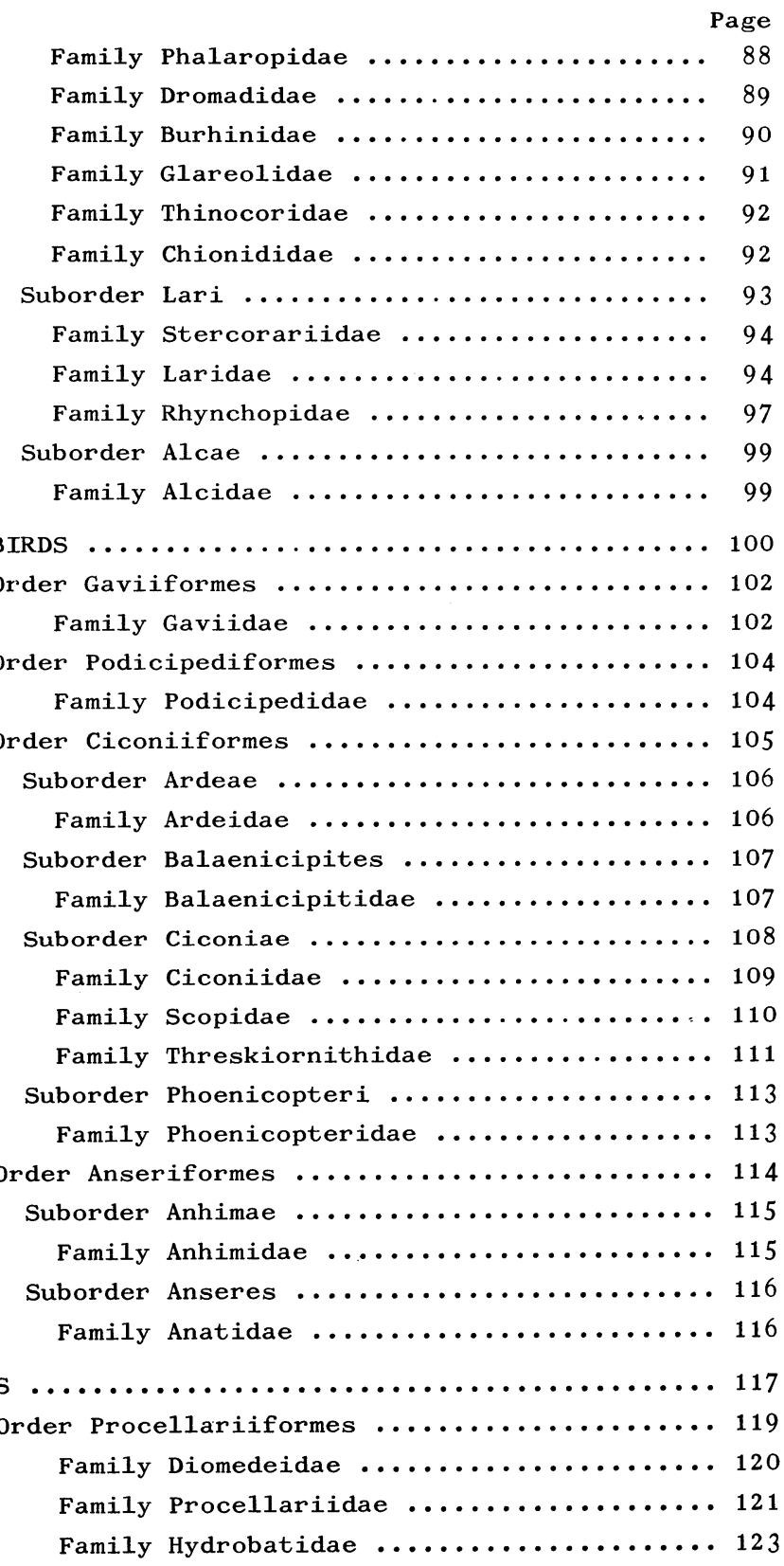




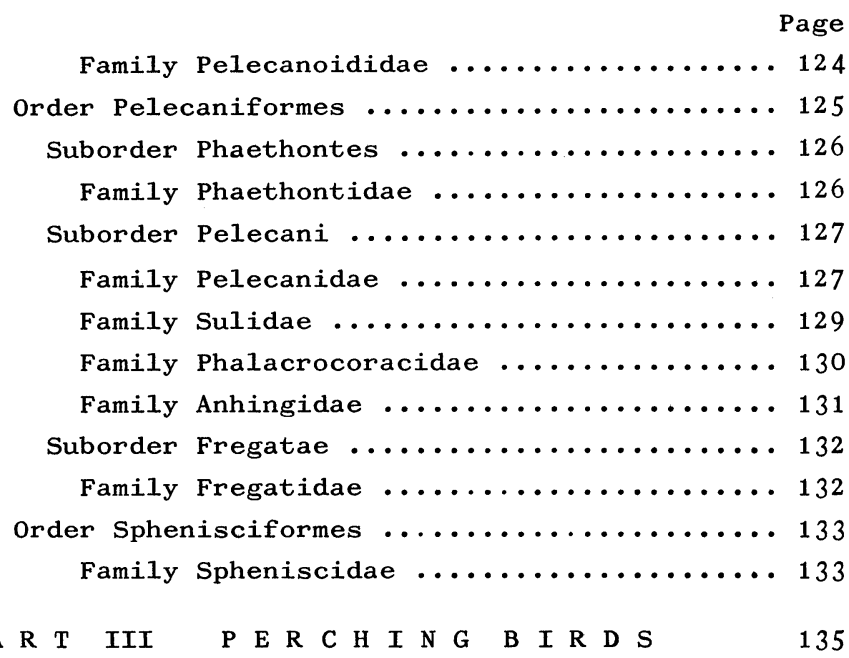

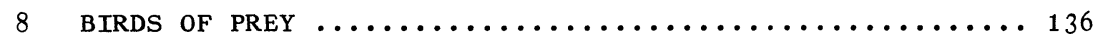

Order Falconiformes .................... 139

Suborder Cathartae ................... 140

Family Cathartidae ................. 140

Suborder Falcones .................... 141

Family Accipitridae ................ 141

Family Falconidae ................. 143

Suborder Sagittarii .................. 144

Family Sagittariidae ................ 144

Order strigiformes .................... 146

Family Tytonidae ................... 146

Family strigidae .................. 147

9 ARBOREAL BIRDS

PIGEONS, PARROTS, CUCKOOS \& COLIES ............... 148

Order Columbiformes ................... 151

Suborder Pterocletes .................. 152

Family Pteroclididae ................ 152

Suborder Columbae ...................... 153

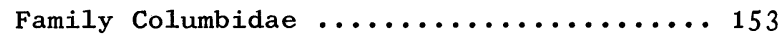

Order Psittaciformes .................. 154

Family Psittacidae ................. 154

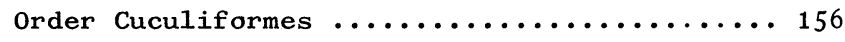

Suborder Cuculi .................... 157 


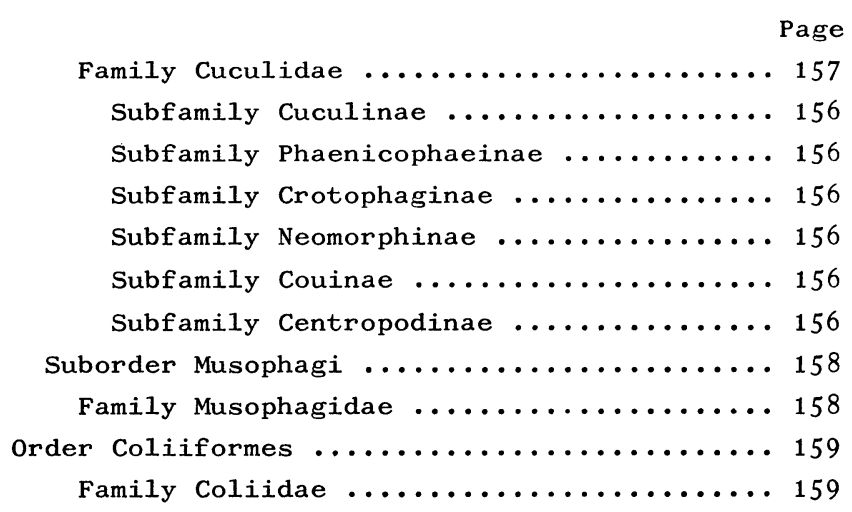

ROLLERS, TROGONS, WOODPECKERS \& ALLIES ............ 160

Order Coraciiformes ................ 162

Suborder Alcedines ................ 163

Family Alcedinidae ................. 164

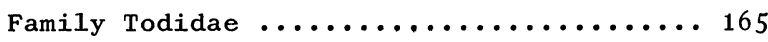

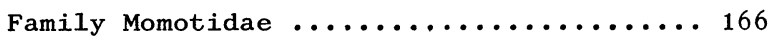

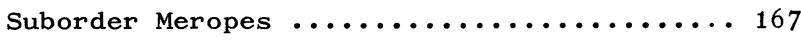

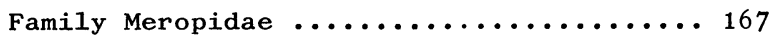

Suborder Coracii .................. 168

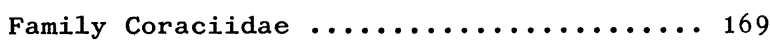

Family Leptosomatidae ............... 170

Family Upupidae ...................... 171

Family Phoeniculidae ......................... 172

Suborder Bucerotes ............... 173

Family Bucerotidae ............... 173

Order Trogoniformes ................ 174

Family Trogonidae ............... 174

Order Piciformes .................. 176

Suborder Galbulae ................ 177

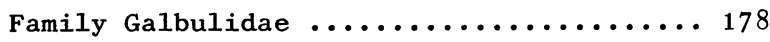

Family Bucconidae .............. 178

Family Capitonidae .................. 180

Family Indicatoridae ............. 182

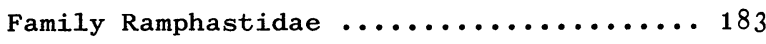

Suborder Pici ................... 184

Family Picidae ..................... 184 


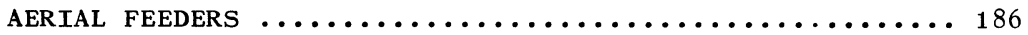

Order Apodiformes ................... 188

Suborder Apodi ..................... 189

Family Apodidae .................. 189

Family Hemiprocnidae ............... 190

Suborder Trochili ................... 191

Family Trochilidae ................ 191

Order Caprimulgiformes ................ 193

Suborder steatornithes ............... 194

Family Steatornithidae ............ 194

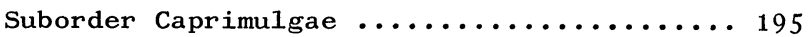

Family Podargidae .................. 195

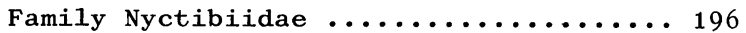

Family Aegothelidae .............. 197

Family Caprimulgidae .............. 198

PRIMITIVE PASSERINES ........................... 199

Order Passeriformes .................. 200

Suborder Eurylaimi .................. 201

Family Eurylaimidae ............... 201

Suborder Tyranni ................... 202

Superfamily Furnarioidea ............ 203

Family Dendrocolaptidae ........... 203

Family Furnariidae .............. 203

Family Formicariidae ............. 203

Family Conopophagidae ............. 204

Family Rhinocryptidae ............. 204

Superfamily Tyrannoidea ............ 205

Family Pittidae .................. 205

Family Philepittidae .................. 205

Family Xenicidae ................ 207

Family Tyrannidae ................ 207

Family Pipridae ................. 207

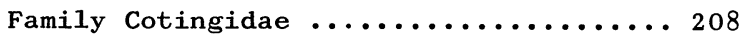

Family Phytotomidae .............. 208

Suborder Menurae .................... 209

Family Menuridae ................ 209

Family Atrichornithidae ........... 210 
THE SONG BIRDS ........................... 211

Suborder oscines ................. 214

Section I The non-arboreal song birds 215

Family Alaudidae .................. 216

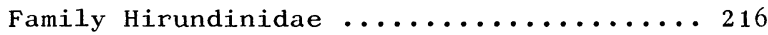

Family Motacillidae ............. 217

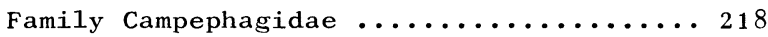

Family Pycnonotidae ................ 219

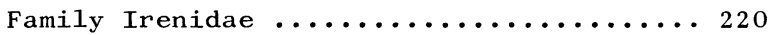

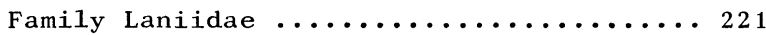

Family Vangidae .................. 222

Family Bombycillidae .............. 223

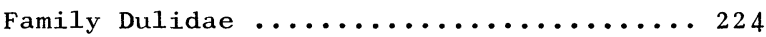

Family Cinclidae ................. 224

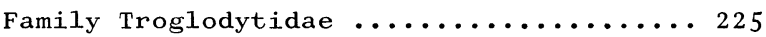

Family Mimidae ................. 226

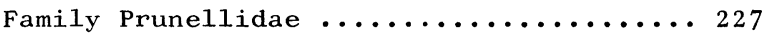

Section II Flycatchers and their allies 228

Family Muscicapidae .............. 228

Subfamily Turdinae .............. 229

Subfamily Timaliinae .............. 231

Subfamily Panurinae (Paradoxornithinae). 233

Subfamily Sylviinae .............. 234

Subfamily Muscicapinae (sensu latu) ...2 235

Subfamily Pachycephalinae .......... 236

Section III The arboreal song birds 237

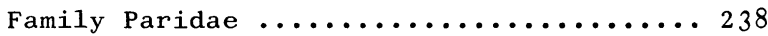

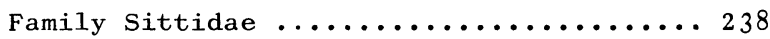

Family Certhiidae .................. 241

Family Climacteridae ............... 241

Family Dicaeidae .................. 242

Family Nectariniidae ................. 243

Family Zosteropidae ................. 244 


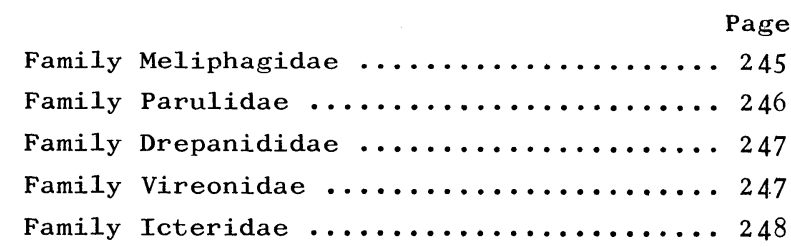

Section IV Song birds which are basically 249 seed-eating

Family Estrildidae ................ 250

Subfamily Estrildinae ............ 249

Subfamily viduinae ............. 249

Family Ploceidae ................ 251

Subfamily Bubalornithinae ......... 249

Subfamily Ploceinae .............. 249

Subfamily Passerinae ............. 249

Family Fringillidae ............. 252

Subfamily Carduelinae ........... 249

Subfamily Fringillinae ............ 249

Subfamily Geospizinae ............ 249

Family Emberizidae .............. 253

Subfamily Pyrrhuloxiinae .......... 249

Subfamily Emberizinae ............. 249

Subfamily Tersininae ............. 249

Subfamily Thraupinae ............. 249

Section V The larger song birds 254

Family Sturnidae .................. 255

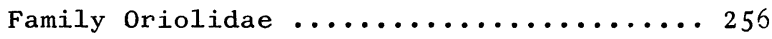

Family Dicruridae ................. 257

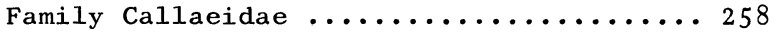

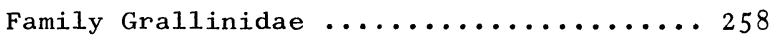

Family Artamidae ................... 259

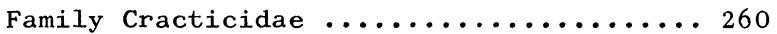

Family Ptilinorhynchidae ........... 261

Family Paradisaeidae .............. 262

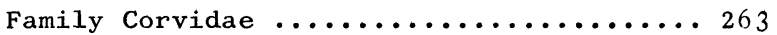

$14 \quad$ GLOSSARY $\ldots \ldots \ldots \ldots \ldots \ldots \ldots \ldots \ldots \ldots \ldots \ldots \ldots \ldots \ldots \ldots \ldots \ldots \ldots$

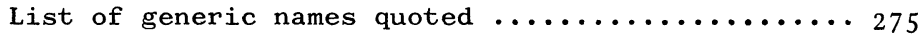

List of common names quoted $\ldots \ldots \ldots \ldots \ldots \ldots \ldots \ldots \ldots$ 